\title{
Comparación de dos protocolos de intervención basados en la Terapia de Aceptación y Compromiso y Entrenamiento en Mindfulness en personas con Trastorno Mental Crónico
}

\author{
Carlos Fco. Salgado Pascual \\ Centro de Psicología PsicACT (Valladolid)
}

\begin{abstract}
ResUmen
Las Terapias Contextuales se están mostrando como una alternativa eficaz en el tratamiento de los problemas de salud mental y en especial de aquellos que implican alto nivel de cronificación. En la presente investigación treinta y seis participantes con importante disfunción social fueron asignados aleatoriamente $a$ tres intervenciones, una basada en la Terapia de Aceptación y Compromiso (ACT), otra basada en el Entrenamiento en Mindfulness (MIND) y un grupo de control en lista de espera que siguió tratamiento habitual (TAU). Se utilizaron medidas de autoinforme y medidas cumplimentadas por personal ajeno y ciego al estudio. Los resultados tanto del grupo ACT como de MIND fueron en la dirección deseada respecto a TAU al post-test. Sin embargo, en los seguimientos, el grupo de ACT obtuvo resultados significativos en la mayoría de las medidas principales del estudio mientras que en el grupo de MIND, los resultados iniciales se fueron diluyendo a lo largo del tiempo. Esta diferente evolución entre grupos necesita de un análisis posterior.
\end{abstract}

Palabras clave: evitación; funcional; aceptación; compromiso; crónico; mindfulness.
ABSTRACT

Contextual Therapies are proving to be an effective alternative in the treatment of mental health problems, especially those involving high level chronicity. In this research thirty-six participants with significant social dysfunction were randomly assigned to three interventions, one based on Acceptance and Commitment Therapy (ACT), one based on Mindfulness training (MIND) and a control group on the waiting list who continued their usual treatment (TAU). Self-report measures and measures completed by anyone and blind to the study personnel were used. The results of both ACT, MIND scored were in the expected direction from TAU at the post-test. In follow-ups, however the ACT group obtained significant results in most of the main measures of the study while in the group of MIND, the initial results were diluting the over time. This different evolution between groups requires further analysis.

Keywords: Avoidance; functional; acceptance; commitment; chronic; mindfulness

Los datos del presente artículo proceden de la Tesis Doctoral del Dr Carlos Salgado dirigida por la Dra. Carmen Luciano y Codirigida por la Dra. Olga Gutiérrez. Parcialmente los datos pertenecientes al grupo ACT fueron publicados con anterioridad en la revista Informaciones Psiquiátricas “Monográfico V Jornadas de Salud Mental y Rehabilitación Psicosocial”, n 213 (4), 2013.

Correspondencia: Dr. Carlos Salgado. Centro de Psicología PsicACT. C/ Londres, 1, Local 1. 47008-Valladolid. Correo electrónico: csalgado@cop.es. 


\section{Introducción}

El Trastorno Mental Grave (TMG) se categoriza formalmente empleando criterios diagnósticos basados en el grado de discapacidad y la presencia de conducta anormal. Se emplean tres criterios: i. Tipo de enfermedad: esquizofrenia y otros tipos de psicosis no orgánica, trastornos graves del estado de ánimo y trastornos de personalidad; ii. Duración de dos años o más del trastorno mental o del tratamiento, y iii. Grado de discapacidad: deterioro moderado en las actividades laborales y no laborales y el deterioro leve en las necesidades básicas (National Institute of Mental Health, 1987).

Fue a partir de 1970 cuando se consideró que las personas con TMG debían recibir tratamiento en lugar de permanecer desatendidas en instituciones por el resto de sus vidas.

Hoy en día, en la mayoría de los países occidentales, las políticas de salud mental fomentan programas psicosociales para las personas que presentan TMG, que están destinadas a proporcionar una vida ordinaria y lo más independiente posible dentro de su comunidad. Aunque estos programas informan de avances sustanciales (véase, Malone, Newron-Howes, Simmonds, Marriot \& Tyrer, 2007), emergen limitaciones importantes como la falta de adherencia al tratamiento y la presencia de recaídas/ readmisiones frecuentes, que precisan de una mayor investigación en el tratamiento del TMG.

Las intervenciones basadas en la aceptación como la Terapia Cognitiva Basada en la Atención Plena (MBCT, Segal, Williams, \& Teasdale, 2002) y la Terapia de Aceptación y Compromiso (ACT, Hayes, Strosahl, \&Wilson, 1999) consti- tuyen un area emergente en psicosis. Mientras que aproximaciones anteriores ponían el énfasis directamente en el cambio de los síntomas cognitivos, MBCT y ACT comparten el objetivo de proveer a los pacientes un modo diferente de relacionarse con sus síntomas psicóticos tales como voces, imágenes o ideas paranoides. Hay, sin embargo, importantes diferencias entre ellas.

En primer lugar, a partir de entrevistas cualitativas realizadas a 16 individuos con psicosis que tomaron parte en un programa de entrenamiento de Mindfulness para pacientes ambulatorios (de ahora en adelante MIND), Abba, Chadwick, \& Stevenson (2008) concluyeron que el MIND ayuda a las personas a reaccionar de diferente manera ante una experiencia psicótica a través de un proceso de tres etapas: prestar atención abiertamente a la experiencia (en lugar de tratar de eliminarla), dejar que la experiencia tenga lugar tal y como es (dejando que los pensamientos y voces vayan y vengan sin reaccionar o revelarse contra ellos) y recuperar la energía a través de la aceptación de la psicosis y mejora del self (en lugar de juzgarse a uno mismo en base a la experiencia psicótica).

Dos estudios controlados han puesto a prueba la eficacia del MIND en psicosis, empleando un diseño aleatorizado de comparación de un grupo que recibió tratamiento frente a un grupo en lista de espera (Chadwick, Hughes, Russell, Russell, \& Dagnan, 2009; Langer, Cangas, Salcedo, \& Fuentes, 2012). Al posttest no se hallaron diferencias significativas en medidas distintas de las habilidades Mindfulness. Es decir, se encontraron diferencias estadísticamente significativas en habilidades de 
Mindfulness, que son precisamente el foco del protocolo MIND, mientras que no se encontró un efecto relevante en el resto de medidas y no se hicieron medidas de seguimiento.

Por otra parte, ACT es una terapia contextual relacionada con la moderna investigación sobre la cognición y el lenguaje propugnada por la Teoría del Marco Relacional (RFT, Hayes, Barnes-Holmes, \& Roche, 2001). La ACT promueve la flexibilidad psicológica enseñando a la persona a actuar de acuerdo a valores personales a pesar de la presencia de experiencias privadas difíciles (Hayes, Luoma, Bond, Masuda, \& Lillis, 2006).

Existen tres estudios controlados aleatorizados que han investigado la aplicación de ACT en psicosis (Bach \& Hayes, 2002; Gaudiano \& Herbert, 2006; White et al., 2011). Bach y Hayes (2002) distribuyeron aleatoriamente a 80 pacientes hospitalizados con síntomas psicóticos positivos en dos grupos: tratamiento regular (TAU) o TAU más cuatro sesiones individuales de $A C T$ (ACT). Aquellos que recibieron el tratamiento ACT, si bien reportaron significativamente una mayor aparición de síntomas, les atribuyeron una menor credibilidad en comparación con aquellos que recibieron el tratamiento TAU. Además, durante los 4 meses de seguimiento la tasa de rehospitalización fue la mitad que la del grupo TAU. Bach, Hayes y Gallop (2012) extendieron los hallazgos obtenidos por Bach y Hayes (2002) a los 4 meses de seguimiento, mostrando que en comparación con TAU, la intervención ACT se asoció con una mayor reducción de la rehospitalización al año de seguimiento. Gaudiano y Herbert (2006) replicaron estos resultados. Cuarenta pacientes con síntomas psicóticos afectivos y no afectivos fueron asignados aleatoriamente a dos tratamientos: tratamiento regular mejorado (ETAU) o el tratamiento regular mejorado con un breve tratamiento en ACT (tres sesiones individuales). El grupo ACT mostró grandes mejoras en síntomas afectivos, funcionamiento social y reducción del estrés asociado a las alucinaciones. Aunque no se registraron diferencias significativas, la tasa de reingreso a los 4 meses fue mayor en el grupo TAU que en el grupo ACT (45\% versus $28 \%$ ). Una tercera investigación (White et al., 2011) realizó el primer ensayo controlado doble ciego sobre ACT en individuos con psicosis. Se asignó aleatoriamente a 27 participantes en dos tratamientos: TAU más diez sesiones individuales de ACT, o simplemente el TAU. Una proporción significativamente mayor de los pacientes de ACT pasó de estar deprimido en la línea base a no estar deprimido a los 3 meses de seguimiento, y las puntuaciones en Evitación Experiencial cambiaron al seguimiento pero no en el post-test. Finalmente, el grupo ACT mostró respecto a TAU un mayor incremento en habilidades de Mindfulness, así como menos síntomas negativos.

Estos estudios han abierto la puerta a la implementación de los tratamientos de Mindfulness y aceptación, sin embargo, hasta ahora ningún estudio aleatorizado ha comparado directamente una intervención basada en ACT y una basada en Mindfulness en psicosis. Con el objetivo de cubrir este vacío, el presente estudio fue diseñado para evaluar los efectos de un protocolo basado en ACT, uno basado en Mindfulness y el tratamiento habitual (TAU). Por otra parte, estos protocolos fueron admi- 
nistrados en formato grupal, en lugar de individual como en los tres estudios anteriores.

Hasta ahora, los estudios en ACT y MIND en psicosis se habían centrado en uno de los tres criterios formales indicados previamente (p.e. diagnóstico formal) para identificar pacientes gravemente enfermos. En el presente estudio, los criterios de inclusión fueron una historia de dos años o más de dificultades en el funcionamiento personal y social, así como un patrón generalizado de Evitación Experiencial o inflexibilidad psicológica. Por otro lado, a diferencia de los estudios previos, centrados en la medida de la severidad de los síntomas como variable dependiente, el presente estudio toma medidas adicionales sobre el funcionamiento diario de los participantes. Además, cabe resaltar que el personal de los centros que recogían algunos de los datos, permanecían ciegos respecto a la condición de tratamiento de los participantes.

A diferencia de los estudios previos de ACT y MIND con un único terapeuta altamente cualificado, en el presente estudio, los protocolos (ACT vs. MIND) fueron desarrollados por cuatro terapeutas (dos para cada protocolo) sin experiencia previa en la aplicación de ambos tipos de terapia, aunque recibieron entrenamiento previo en el tratamiento correspondiente. Además, los terapeutas fueron asignados de manera aleatoria a cada condición con el objetivo de evitar la adhesión entre tratamiento y terapeuta.

Dado el apoyo empírico de la ACT con pacientes con psicosis, así como el limitado pero esperanzador apoyo de los datos a la eficacia del entrenamiento en Mindfulness, no pre- decimos diferencias en la efectividad total de ambos protocolos en el post-test. Sin embargo, dada la evidencia obtenida en algunos de los estudios de ACT para psicosis respecto a que los efectos de dicha intervención pueden observarse más a largo plazo, hipotetizamos que de acuerdo con el énfasis de la ACT en los valores, las diferencias entre ambos tratamiento y los tamaños del efecto serían mayores en el periodo de seguimiento.

\section{Método}

Participantes

Los participantes fueron seleccionados de tres centros pertenecientes a una entidad privada de salud mental de Valladolid (España). Los criterios de inclusión fueron dos dimensiones comunes (discapacidad y duración) empleadas para identificar pacientes con trastornos mentales graves. El criterio de discapacidad se definió como el deterioro en el funcionamiento en múltiples areas, apoyado por una puntuación superior a 40 en el Global Assessment Functioning Scale (GAF) (Endicott, Spitzer, Fleiss, \& Cohen, 1976). El criterio de duración se definió como dos ó más años de diagnóstico de enfermedad mental o tratamiento. Por otra parte, para asegurar que los participantes tenían un patrón de evitación experiencial clínicamente significativo, solo se incluyeron personas cuyas puntuaciones en el Acceptance and Action Questionnaire (AAQ-II) (Bond et al., 2011) en el pre-test fueran superiores a 48 y/o si se identificaban en la entrevista inicial problemas graves relacionados con la regulación de las experiencias privadas. Los criterios de exclusión fueron i. la presencia de 
diagnóstico primario de demencia o discapacidad intelectual significativa; ii una incapacidad para proporcionar el consentimiento informado. Las características clínicas y demográficas de los participantes de cada condición aparecen reflejadas en la Tabla 1.

La muestra final del estudio incluyó 36 participantes (16 mujeres, 20 hombres) de edades entre 20 y 80 años ( $M=49.56, S D=13.83)$. Todos los participantes estaban desempleados debido a sus condiciones psiquiátricas. La mayoría de ellos (61\%) residían en el hospital psiquiátrico, y la media del periodo de hospitalización era superior a cinco años (68,92 meses). El resto vivía con sus familias (33\%), en una comunidad religiosa (3\%), o con su pareja (3\%).
La mayoría de los participantes no tomaban drogas (94\%), mientras que el 3\% consumía alcohol y el 3\% otras sustancias (sólo uno de los participantes cumplió criterios de dependencia a sustancias). Los pacientes mostraron un alto nivel de malestar psiquiátrico y pobre funcionamiento social. A pesar de que los criterios de inclusión se centraron en características funcionales (i.e., cronicidad, discapacidad, evitación experiencial) y no en el diagnóstico en sí, los diagnósticos formales se tuvieron en cuenta a la hora de equilibrar la distribución de los pacientes en cada uno de los tratamientos (ACT, MIND, TAU) (ver Tabla 1). Al principio del estudio todos los participantes tomaban medicación prescrita por su psiquiatra, excepto

Tabla 1

Características clínicas y demográficas de la muestra

\begin{tabular}{|c|c|c|c|}
\hline \multirow[b]{2}{*}{ Variables } & \multicolumn{3}{|c|}{ Condición } \\
\hline & $\mathrm{ACT}$ & MIND & TAU \\
\hline Edad (media) & 51,92 & 47,38 & 49,55 \\
\hline Tiempo de diagnóstico (años) & 19,55 & 16,17 & 25,36 \\
\hline Mujeres & $50 \%(6)$ & $38,5(5)$ & $45,5(5)$ \\
\hline Hombres & $50 \%(6)$ & $61,5(8)$ & $54,5(6)$ \\
\hline Tiempo de hospitalización (meses) & 86,78 & 52,90 & 68,86 \\
\hline \multicolumn{4}{|l|}{ Diagnóstico } \\
\hline Esquizofrenia & $4(33,00 \%)$ & $4(30,80 \%)$ & $5(45,45 \%)$ \\
\hline Trastorno bipolar & $1(8,30 \%)$ & $2(15,40 \%)$ & $0(0,00 \%)$ \\
\hline Psicosis inespecífica no orgánica & $1(8,30 \%)$ & $0(0,00 \%)$ & $2(18,18 \%)$ \\
\hline Trastorno Depresivo recurrente & $1(8,30 \%)$ & $1(7,70 \%)$ & $1(9,09 \%)$ \\
\hline Trastorno Somatomorfo indiferenciado & $0(0,00 \%)$ & $1(7,70 \%)$ & $0(0,00 \%)$ \\
\hline Trastorno esquizoafectivo & $1(8,30 \%)$ & $2(15,40 \%)$ & $1(9,09 \%)$ \\
\hline Trastorno de personalidad & $3(25,00 \%)$ & $2(15,40 \%)$ & $2(18,18 \%)$ \\
\hline Dependencia de drogas & $1(8,30 \%)$ & $0(0,00 \%)$ & $0(0,00 \%)$ \\
\hline Ciclotimia & $0(0,00 \%)$ & $1(7,70 \%)$ & $0(0,00 \%)$ \\
\hline Total & 12 & 13 & 11 \\
\hline
\end{tabular}


dos participantes en la condición ACT, uno en la condición MIND y uno en la condición TAU. Finalmente, aunque todos los participantes informaron haber tenido una larga historia de tratamientos tanto psiquiátricos como psicológicos, no hubo acceso a información detallada acerca del tipo de tratamientos psicológicos recibidos previamente.

El flujo de participantes a lo largo del estudio se resume en la Figura 1.

\section{Medidas Basadas en Autoinformes}

Flexibilidad psicológica (AAQ-II)

Para medir la flexibilidad psicológica se empleó la segunda versión del Cuestionario de Aceptación y Acción (AAQ-II, Bond et al., 2011), tomando su adaptación española (Ruiz, Langer, Herrera, Luciano, Cangas, \&Beltran, 2013). Eneste cuestionario, las respuestas se valoran en una escala tipo Likert con 7 opciones de respuesta desde 1 (nunca cierto) hasta 7 (siempre cierto).

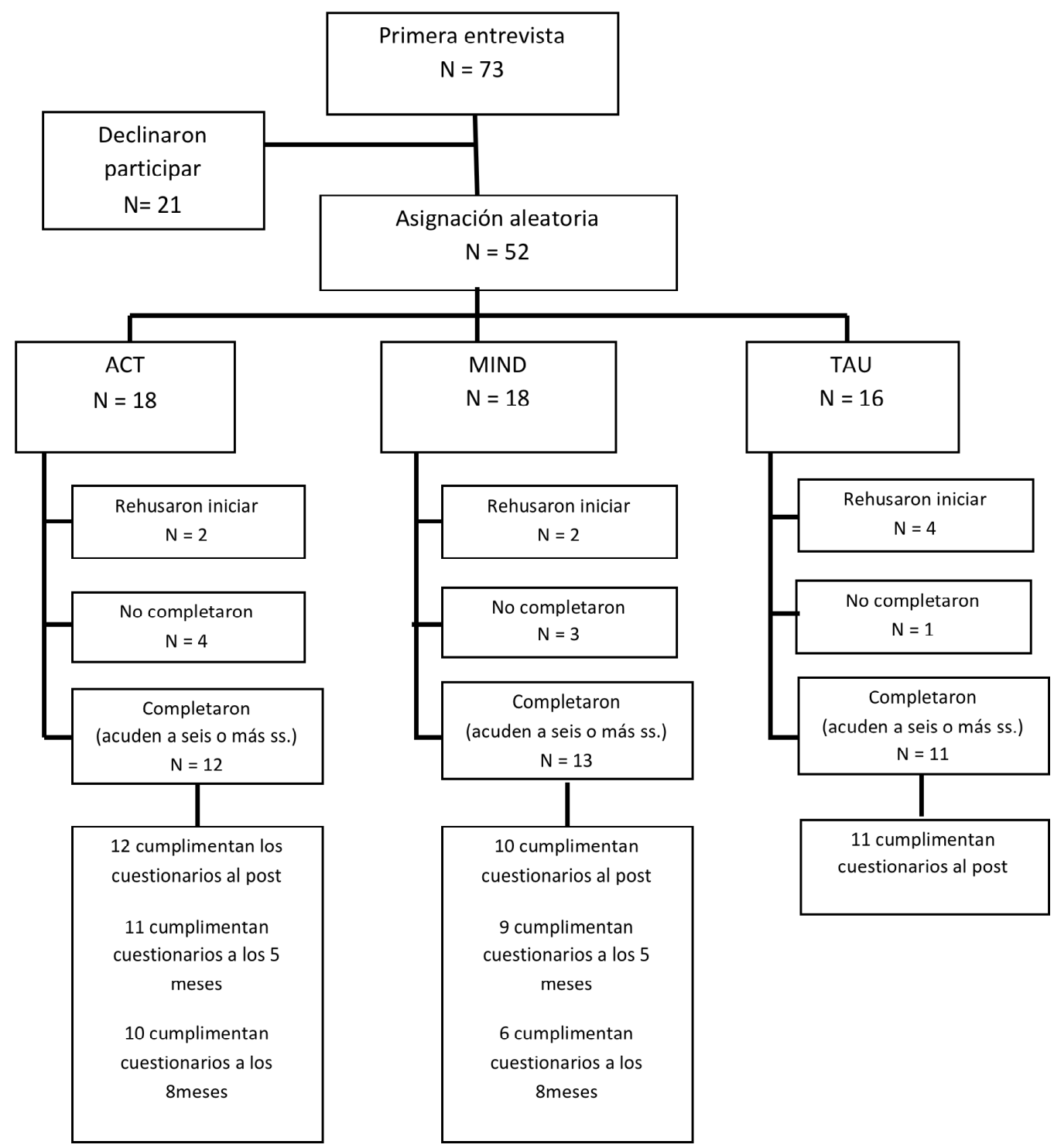

Figura 1. Diagrama de flujo de los participantes. 
Las puntuaciones totales van desde 10 hasta 70 , indicando las puntuaciones mayores un mayor nivel de evitación experiencial y, por tanto, menores niveles de flexibilidad psicológica. La media de la muestra normativa es de 18.51 puntos (SD = 7.05) (Bond et al., 2011). Estudios previos indican una satisfactoria consistencia interna, siendo la media del coeficiente alfa .84, y la fiabilidad de los test-retest a los 3 y 12 meses de .81 y .79, respectivamente (Bond et al., 2011).

\section{Síntomas Psicopatológicos (SA-45)}

El cuestionario de evaluación de 45 síntomas (SA-45, Davison et al., 1997) es una medida de síntomas psicopatológicos derivada del Symptom Checklist-90-R (SCL-90-R, Derogatis, 1994). El SA-45 consiste en nueve subescalas que evalúan nueve dominios sintomáticos. Emplea un formato de escala tipo Likert, conteniendo cada subescala cinco items que puntúan desde cero (nada en absoluto) hasta 4 (extremadamente). En cada subescala, las puntuaciones totales van de cero a veinte, indicando mayores puntuaciones una mayor severidad de la sintomatología psiquiátrica.

En el presente estudio, se emplearon cuatro subescalas (depresión, ansiedad, ideación paranoide y psicosis) de la versión española (Sandín, Valiente, Chorot, Santed, \& Lostao, 2008), dado que estas subescalas se consideraron medidas adecuadas para la población de pacientes con trastornos mentales graves y crónicos. Las puntuaciones medias para estas subescalas en una muestra normativa son 5.32 ( $\mathrm{SD}=4.4)$ en depresión, $3.56(\mathrm{SD}=3.8)$ en ansiedad, 4.53 (SD = 3.7) en ideación paranoide y 1.7 (SD = 2.2) en psicosis (Sandín et al., 2008).

\section{Autorregistro de actividad (AAC)}

Los participantes fueron requeridos para que completasen un diario en el cual registrasen las actividades en dirección de valor realizadas en la semana previa a las sesiones de evaluación.

Registros completados por el personal hospitalario.

Los miembros del personal sanitario que estaban encargados de la cumplimentación de estas medidas, no conocían la condición de tratamiento a la que pertenecía cada participante.

\section{Informe de actividad (IAC)}

Un miembro del personal valoraba a través de una Escala de Analogía Visual (VAS) de 0 (ninguna actividad) a 10 (mucha actividad), el nivel de actividad dirigida a objetivos observado en los participantes durante la semana previa.

\section{Terapeutas}

Las terapeutas fueron cuatro mujeres (edad media $=28.4 ; S D=2.19)$ que habían completado recientemente la Licenciatura en Psicología y habían realizado un Máster en Psicología Clínica en el momento del estudio. Las cuatro eran terapeutas noveles, sin experiencia anterior en la aplicación de ACT o MIND. Las terapeutas fueron asignadas aleatoriamente a uno de los dos tratamientos: dos recibieron formación en ACT y otras dos en MIND. Todas participaron en el entrenamiento realizado por el autor, que tenía alta formación y varios años de experiencia en la aplicación tanto de ACT como de MIND. EI entrenamiento consistió durante nueve meses, en una sesión semanal de dos horas y media de 
duración e incluyó una combinación de seminarios, modelado, role-plays, lecturas obligatorias y ejercicios para casa.

El periodo de formación empezó para ambos grupos con ocho horas de clases sobre principios de la conducta y generalidades de las terapias contextuales o de tercera ola. A continuación se impartieron 20 horas de seminarios específicos en MIND o ACT respectivamente, y 40 horas de entrenamiento empleando role-playing y ejemplos de casos. Después, se grabó en video la actuación de las terapeutas empleando MIND o ACT. Dicha grabación fue revisada por expertos independientes que proporcionaron feedback acerca de la necesidad o no de más práctica para las terapeutas. Finalmente tuvieron $25 \mathrm{~h}$ adicionales de entrenamiento (un taller práctico, así como trabajo específico en el protocolo a administrar durante el estudio).

\section{Condiciones experimentales}

Tratamiento habitual (TAU)

TAU consistió en la administración de psicofármacos y la asistencia a sesiones grupales de rehabilitación psicosocial (i.e., psicoeducación, habilidades sociales, terapia ocupacional). Estas actividades las llevaron a cabo miembros del personal sanitario que no tenían conocimiento del estudio.

A los pacientes de TAU se les comunicó que estaban en un programa de lista de espera, si bien, no se pudo mantener dicha condición en los seguimientos (a los 5 y 8 meses) por la imposibilidad de extender por más tiempo la colaboración de las terapeutas. Por este motivo, a la finalización de la aplicación de los protocolos, este grupo recibió el protocolo basado en ACT por consideralo el más completo inicialmente.

$\underline{A C T}+$ TAU (para evitar confusión, en adelante se etiquetará simplemente como ACT)

Todos los participantes de este grupo recibieron el tratamiento regular (TAU). El protocolo de ACT consistió en siete sesiones semanales de hora y media de duración (dos sesiones iniciales con formato individual y cinco con formato grupal) dirigidas por dos de las terapeutas. El protocolo de ACT se basó en los manuales de ACT de Wilson y Luciano (2002) y Hayes y Smith (2005), con metáforas y ejercicios específicos que fueron adaptados a la población.

Se siguió un formato individual en las dos primeras sesiones para centrar el protocolo en el análisis funcional de las conductas del paciente así como para generar la experiencia de desesperanza creativa, identificar las trayectorias personales de valor y los pensamientos, sentimientos y reglas que funcionaban como barreras para realizar acciones valoradas. Las sesiones individuales permitieron cuadrar los métodos de tratamiento a nivel grupal para que fueran individualmente productivos. Así, los ejercicios y metáforas de clarificación de valores y objetivos se emplearon para identificar pequeños pasos en dirección de valor y el papel que tenían los pensamientos/ emociones displacenteros (tal como la rabia o el retraimiento social) a la hora de realizar acciones inconsistentes con sus valores. Por ejemplo, se animó a los participantes a comprometerse con una acción específica que pudiera ha- 
cerse dentro y fuera del espacio de la terapia para cultivar la dirección elegida. Además, se les pidió que realizaran ejercicios con los ojos cerrados para que notaran y observaran los eventos privados derivados de imaginarse a sí mismos realizando la acción a la que se habían comprometido, asociada con el valor elegido. Finalmente, se instó a los participantes a llevar a cabo sus compromisos (hasta la siguiente sesión grupal) como parte de la práctica de aceptación de las barreras, en dirección a alimentar los valores elegidos.

Después de la segunda sesión individual, se llevaron a cabo cinco sesiones grupales. Cada una de estas sesiones comenzó con una revisión de las actividades realizadas por los participantes la semana anterior y de las barreras identificadas cuando no se cumplieron los compromisos. A continuación, se introdujeron los métodos experienciales centrales a trabajar en cada una de las sesiones. Cada sesión grupal terminaba con dos intervenciones: (a) un resumen del significado del trabajo realizado en sesión y los compromisos a llevar a cabo antes de la siguiente y (b) un ejercicio de defusión centrado en la práctica de ser consciente del flujo de eventos privados sin engancharse a ellos y el significado de estos ejercicios a la hora de avanzar en la vida. Las tres primeras sesiones grupales (sesión 3,4 y 5) se focalizaron en los esfuerzos previos de los participantes por eliminar el malestar y en las consecuencias negativas de dichas conductas, explorando cómo tratar de controlar las experiencias mentales complicadas puede ser parte del problema más que la solución. Por otro lado, se incluyeron ejercicios expe- rienciales para practicar la aceptación y trabajar habilidades de defusión. La cuarta sesion grupal (sesión 6) se centró en promover las experiencas del "yo como contexto" a través de ejercicios experienciales que enfatizan la discriminación de la experiencia de uno mismo notando los pensamientos y sentimientos que aparecen en el momento presente. La última sesión grupal se empleó para promover de nuevo la aceptación y las habilidades de defusión añadiendo más ejercicios experienciales en conexión con las actividades elegidas por los participantes.

Se llevaron a cabo tres sesiones de refuerzo a las 2, 6 y 12 semanas después de la última sesión grupal. Éstas se centraron en revisar las acciones acordes con los valores personales; explorar los pensamientos y sentimientos difíciles que aparecen a lo largo del camino y practicar las habilidades de defusión y aceptación. A pesar de existir un detallado protocolo para cada sesión, se introdujeron breves interacciones si los participantes se fusionaban con pensamientos o emociones surgidos durante la sesión. En esos momentos, las terapeutas ayudaban a poner la atención en el momento presente, a notar que esos eventos privados eran parte de la persona en el presente y practicaban una nueva forma de regulación. También se enfatizó el papel de las recaídas como parte del proceso terapéutico.

Se hicieron esfuerzos para asegurar la fidelidad al tratamiento. Después de cada sesión, las terapeutas marcaban en una lista los elementos que habían usado, así como los componentes incluídos durante la sesión para evaluar la adherencia al protocolo. 
$\underline{\text { MIND + TAU (a partir de este momento }}$ MIND).

El protocol MIND también consistió en siete sesiones semanales de hora y media de duración, dirigidas por las otras dos terapeutas. El protocolo MIND se basó en los trabajos de Kabat-Zinn (1990; 2001; 2009) así como en los procedimientos de la Terapia Cognitiva Basada en Mindfulness (MBCT) para la depresión de Segal et al. (2002). Dado que el protocolo MIND no incluye atención individual específica relacionada con valores, tal y como ocurre en el caso de ACT, todas las sesiones tuvieron un formato grupal.

Cada sesión comenzó con una breve revisión de las actividades de la semana anterior y un breve ejercicio práctico de Mindfulness. A continuación, se incluían los ejercicios centrales de la sesión. Al final de cada sesión se resumían los ejercicios fundamentales y se hacía la propuesta de las actividades a realizar hasta la semana siguiente. Finalmente se realizaba de nuevo un breve ejercicio de Mindfulness.

La sesión 1 tenía un formato psicoeducativo y se centró en la explicación del funcionamiento del "piloto automático" para introducir, posteriormente, los principios y actitudes básicas de la práctica de Mindfulness. Por otro lado, se realizaron múltiples ejercicios breves para facilitar una primera experiencia con Mindfulness, para acabar con el ejercicio del escáner corporal (o Body Scan). La sesión 2 se dedicó a asentar la atención sobre la respiración a través de múltiples ejercicios. Se exploraron las sensaciones durante la respiración mientras se notaban los pensamientos y sentimientos difíciles que pudieran aparecer y tomando distancia res- pecto a ellos. De esta forma se trabajó el notar las distracciones y conscientemente centrar la atención de nuevo en la respiración. La sesión 3 se centró en notar la experiencia corporal de caminar así como de otros movimientos, empleando ejercicios del "Hatha Yoga" que fueron modificados y adaptados específicamente para este protocolo. La sesión 4 tuvo como objetivo generalizar la práctica de la atención a la respiración a situaciones de la vida diaria. Para ello se presentó el ejercicio "espacio para la repiración respiración de tres minutos". De la misma forma, la práctica del paseo consciente se propuso para fomentar la generalización de la atención plena en movimiento fuera del contexto del aula. La sesión 5 se centró en la práctica del "Mindfulness de la postura, la respiración, los sonidos y los pensamientos" con el propósito de aprender no sólo a centrar la atención en un punto, sino también a mover el foco a diferentes elementos. En la sesión 6 se centró la práctica en ejercicios de Mindfulness dirigiendo la atención hacia pensamientos y emociones displacenteras con el propósito de aprender a vivir con la frustración y otras experiencias desagradables. En la última sesión (sesión 7), se invitó a los participantes a realizar de nuevo el escáner corporal (Body Scan) con el objetivo de comparar esta última experiencia con la que tuvieron en la primera sesión. Por otro lado, se recordó la importancia de la práctica regular de Mindfulness a lo largo del resto de la vida.

Al igual que en la condición ACT, se incluyeron tres sesiones de refuerzo a las 2,6 y 12 semanas después de la última sesión grupal. Durante cada una de estas sesiones, se regis- 
tró la práctica realizada por cada paciente y se atendió a las dudas, dificultades y obstáculos experimentados. Se añadieron prácticas adicionales de Mindfulness en el grupo.

Al igual que en la condición ACT, en el protocolo MIND los participantes continuaron recibiendo su tratamiento regular (TAU). De manera similar, los terapeutas MIND registraron en una lista aquellos métodos que habían empleado durante cada sesión para evaluar la adherencia al protocolo.

\section{Procedimiento}

El Comité de Ética del Centro Clínico de referencia informó positivamente sobre la realización del estudio. A continuación, el primer autor se reunió individualmente con los participantes de cada localidad para explicar el programa. Entonces, se pidió a los participantes que firmaran el consentimiento informado $y$ respondieran a los cuestionarios de evaluación (evaluaciones pre-test). También se pidió al personal hospitalario que tomase las medidas relacionadas con los participantes (ver la sección Medidas en el apartado Método). Una vez todas las medidas pre-test se hubieron completado, aquellos participantes $(\mathrm{N}=52)$ que se mostraron dispuestos a continuar fueron asignados de manera aleatoria (usando un método de randomización computerizado) a una de las tres condiciones experimentales.

Ocho participantes se retiraron en el primer momento sin llegar a iniciar la intervención. Así pues, cada condición experimental tuvo un total de 16, 16 y 12 participantes, respectivamente en las condiciones ACT, MIND y TAU (ver Figura 1, diagrama de flujo de participantes).
En cada localidad había un grupo ACT otro MIND y otro TAU, resultando en cada grupo de tres a seis participantes.

Todas las sesiones de cada condición experimental estuvieron a cargo de dos terapeutas previamente entrenadas en su respectivo protocolo, una de las terapeutas conducía la sesión y la otra ayudaba como coterapeuta. Para minimizar el error debido al terapeuta, ambas llevaron a cabo los dos roles a lo largo de las sesiones y se contrabalancearon respecto a las sesiones y localidades (por ejemplo: en la sesión 1 hacía de terapeuta en la localidad A y C, y de coterapeuta en localidad B. En la sesión 2 hacía de terapeuta en la localidad B, y de coterapeuta en $\mathrm{A}$ y $\mathrm{C}$ ).

El protocolo se desarrolló a lo largo de siete sesiones de aproximadamente 90 minutos, y una frecuencia semanal. Las correspondientes sesiones de ambos protocolos fueron aplicadas durante la misma semana en cada localización (por ejemplo: semana 1, sesión 1: lunes-localización $A$, miércoles-localización $B$ y viernes-localización C). El criterio para considerar el tratamiento como completado fue haber asistido a seis de las siete sesiones.

Una vez finalizada la implantación de los protocolos, los participantes de las tres condiciones experimentales respondieron a los autoinformes. Además, el personal hospitalario recogió las medidas indicadas en la sección anterior.

Los participantes de ACT y MIND recibieron tres sesiones adicionales de refuerzo (a las 2, 6 y 12 semanas después de la última sesión grupal). Finalmente, los participantes de MIND y ACT completaron evaluaciones de seguimiento a los 5, y 8 meses después de la última se- 
sión grupal. La Figura 1 muestra los participantes que completaron el tratamiento.

\section{Resultados}

Análisis estadístico

Se analizaron las principales variables de resultado empleando análisis de medidas repetidas con participantes anidados dentro de las tres condiciones experimentales (ACT, MIND y TAU) y los dos momentos de evaluación (pre y post). Para comparar los grupos MIND y ACT, se emplearon dos condiciones de tratamiento y cuatro momentos de evaluación. De esta manera, los análisis se centraron en las interacciones Momento x Condición de tratamiento y en el patrón de diferencias intragrupales del pre al post-tratamiento, y a los seguimientos (5 y 8 meses). La significación clínica se analizó empleando el estadístico "d" de Cohen. Los tamaños del efecto $.2, .5$ y .8 se consideraron pequeño, moderado y grande respectivamente.

\section{Medidas previas al tratamiento}

No hubo diferencias significativas entre las condiciones en las medidas previas al tratamiento $(p>.050)$.

\section{Desgaste}

El desgaste no varió significativamente entre grupos ( $p>.050$ ). En el grupo ACT, el $25 \%$ (4 de 16 pacientes) no completó el protocolo (no acudió a seis o más sesiones), mientras que en la condición MIND fue del 18,75\% (3 de 16), y de $8,33 \%$ ( 1 de 12 ) en el grupo TAU.

En el seguimiento llevado a cabo a los 8 meses, el $16,67 \%$ de los pacientes que completaron el protocolo ACT se habían retirado del estudio ( 2 de 12), comparado con el 53,85\% (7 de 13) de los participantes que completaron el protocolo MIND. Esta diferencia no resultó significativa ( $p>$.050). Los pacientes que se retiraron del estudio no diferían significativamente en ninguna de las medidas de los participantes que no se retiraron.

\section{Adherencia al protocolo}

La adherencia al protocolo se calculó analizando la correspondencia entre los elementos de cada protocolo y los elementos identificados por los terapeutas durante cada sesión. No hubo diferencias en adherencia al protocolo entre las condiciones ACT y MIND ( $p>.050)$.

Entre las terapeutas ACT hubo una correspondencia del $74 \%$ y del $81 \%$, mientras que las terapeutas MIND del $84 \%$ y del $86 \%$. Una terapeuta MIND no alcanzó el criterio de al menos un $70 \%$ de adherencia en las sesiones 2 y 6 , mientras que una terapeuta ACT no alcanzó el criterio en la sesión 3 y la otra en la sesión 6.

\section{Medidas de resultado primarias}

La Tabla 2 y la Figura 2 muestran los estadísticos descriptivos para las medidas de respuesta primarias para los tres grupos.

\section{Flexibilidad psicológica (AAQ-II)}

La Tabla 2 y la Figura 2 muestran que entre el pre-test y el post-test, el grupo TAU no mostró diferencias significativas $(p>.050)$. En el grupo MIND, las puntuaciones disminuyeron entre el pre-test y el post-test, y empezó a aumentar ligeramente después del post-test. Sin embargo, estos cambios no resultaron estadísticamente significativos $(p>.050)$. 
Tabla 2

Medias y desviaciones típicas

\begin{tabular}{|c|c|c|c|c|c|c|c|c|c|c|}
\hline & ACT & & & & MIND & & & & TAU & \\
\hline & Pre & Post & $5 m$ & $8 m$ & Pre & Post & $5 m$ & $8 m$ & Pre & Post \\
\hline AAQ-II & $\begin{array}{c}46.75 \\
(11.06)\end{array}$ & $\begin{array}{c}42.67 \\
(12.22)\end{array}$ & $\begin{array}{c}36.20 \\
(12.19)\end{array}$ & $\begin{array}{l}31.50 \\
(8.54)\end{array}$ & $\begin{array}{c}44.90 \\
(13.59)\end{array}$ & $\begin{array}{c}35.30 \\
(13.02)\end{array}$ & $\begin{array}{c}36.83 \\
(12.19)\end{array}$ & $\begin{array}{c}41.00 \\
(18.27)\end{array}$ & $\begin{array}{l}45.73 \\
(9.85)\end{array}$ & $\begin{array}{c}45.27 \\
(12.11)\end{array}$ \\
\hline AAC & $\begin{array}{c}4.00 \\
(2.49)\end{array}$ & $\begin{array}{c}5.73 \\
(3.26)\end{array}$ & $\begin{array}{c}6.50 \\
(2.88)\end{array}$ & $\begin{array}{c}6.45 \\
(2.88)\end{array}$ & $\begin{array}{c}4.23 \\
(2.17)\end{array}$ & $\begin{array}{c}5.54 \\
(2.11)\end{array}$ & $\begin{array}{c}6.29 \\
(2.43)\end{array}$ & $\begin{array}{c}5.17 \\
(2.79)\end{array}$ & $\begin{array}{c}4.55 \\
(2.16)\end{array}$ & $\begin{array}{c}4.70 \\
(2.31)\end{array}$ \\
\hline IAC & $\begin{array}{c}5.63 \\
(1.06)\end{array}$ & $\begin{array}{c}6.00 \\
(1.41)\end{array}$ & $\begin{array}{l}6.71 \\
(.76)\end{array}$ & $\begin{array}{l}7.00 \\
(.82)\end{array}$ & $\begin{array}{c}6.42 \\
(1.38)\end{array}$ & $\begin{array}{l}7.36 \\
(.92)\end{array}$ & $\begin{array}{c}7.18( \\
.87)\end{array}$ & $\begin{array}{c}7.00 \\
(1.41)\end{array}$ & $\begin{array}{c}5.80 \\
(1.32)\end{array}$ & $\begin{array}{c}5.72 \\
(1.42)\end{array}$ \\
\hline SA-45 Dep & $\begin{array}{c}9.90 \\
(5.38)\end{array}$ & - & - & $\begin{array}{c}5.40 \\
(4.06)\end{array}$ & $\begin{array}{c}7.14 \\
(6.34)\end{array}$ & - & - & $\begin{array}{c}9.33 \\
(6.74)\end{array}$ & $\begin{array}{c}8.78 \\
(5.95)\end{array}$ & - \\
\hline SA-45 Ans & $\begin{array}{c}8.40 \\
(5.87)\end{array}$ & - & - & $\begin{array}{c}4.60 \\
(4.14)\end{array}$ & $\begin{array}{c}7.86 \\
(4.71)\end{array}$ & - & - & $\begin{array}{c}7.33 \\
(5.57)\end{array}$ & $\begin{array}{c}7.89 \\
(2.97)\end{array}$ & - \\
\hline $\begin{array}{c}\text { SA-45 Id } \\
\text { Par }\end{array}$ & $\begin{array}{c}8.70 \\
(4.14)\end{array}$ & - & - & $\begin{array}{c}4.80 \\
(2.10)\end{array}$ & $\begin{array}{c}5.57 \\
(3.87)\end{array}$ & - & - & $\begin{array}{c}6.83 \\
(3.37)\end{array}$ & $\begin{array}{c}7.22 \\
(5.59)\end{array}$ & - \\
\hline $\begin{array}{c}\text { SA-45 } \\
\text { Psic }\end{array}$ & $\begin{array}{c}3.80 \\
(5.31)\end{array}$ & - & - & $\begin{array}{c}2.30 \\
(3.34)\end{array}$ & $\begin{array}{c}1.29 \\
(1.98)\end{array}$ & - & - & $\begin{array}{c}3.17 \\
(5.42)\end{array}$ & $\begin{array}{c}4.89 \\
(3.37)\end{array}$ & - \\
\hline
\end{tabular}

Nota: $5 \mathrm{~m}=$ Seguimiento a los 5 meses $; 8 \mathrm{~m}=$ Seguimiento a los 8 meses; AAQ-II = Acceptance and Action Questionnaire (rango: 10-70); $A A C=$ Autorregistro de actividad $; I A C=$ Informe de actividad del personal hospitalario (rango: 0-10);SA-45 Dep = Subescala de depresión del SA-45; SA-45 (rango: 0-20); Ans = Subescala de ansiedad del SA-45 (rango: 0-20); SA-45; Id Par = Subescala Ideación Paranoide del SA-45 (rango: 0-20); SA-45; Psic = Subescala psicoticismo del SA-45 (rango: 0-20). Las diferencias significativas respecto al Pre-test $\mathrm{p}<.05$ se muestran en sombreado.

AAQ II

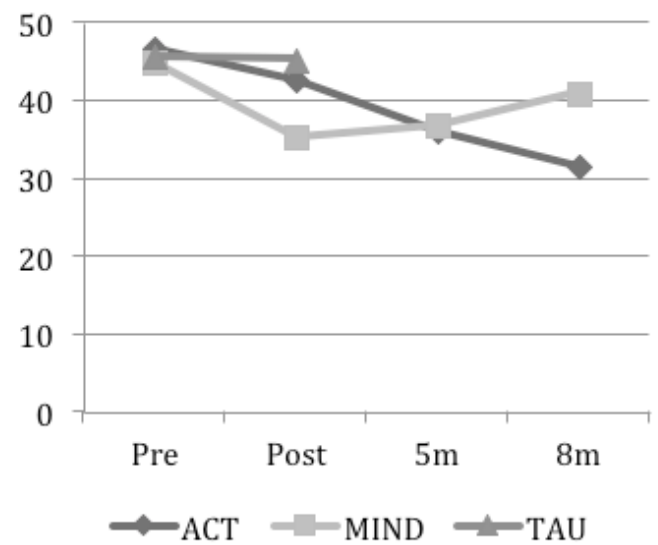

AAc

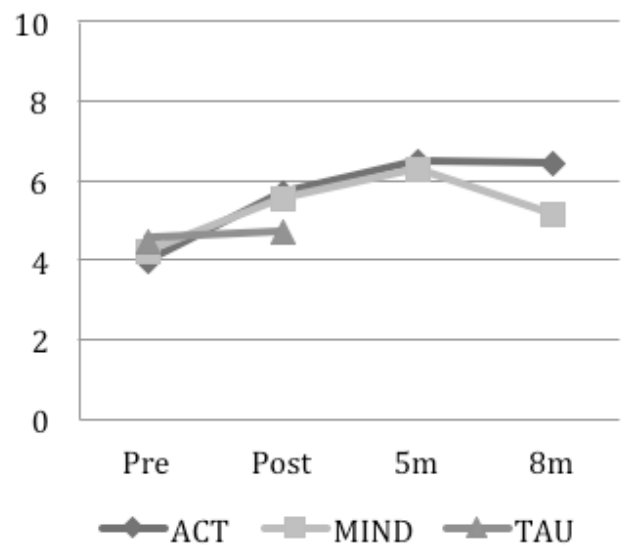


IAC

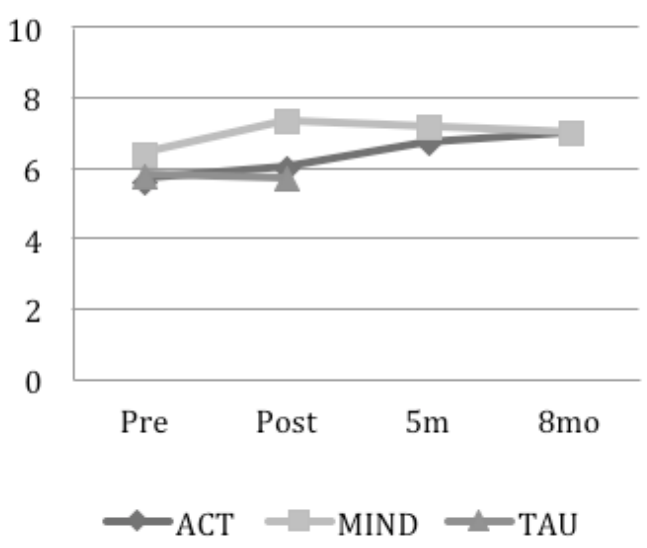

Ansiedad

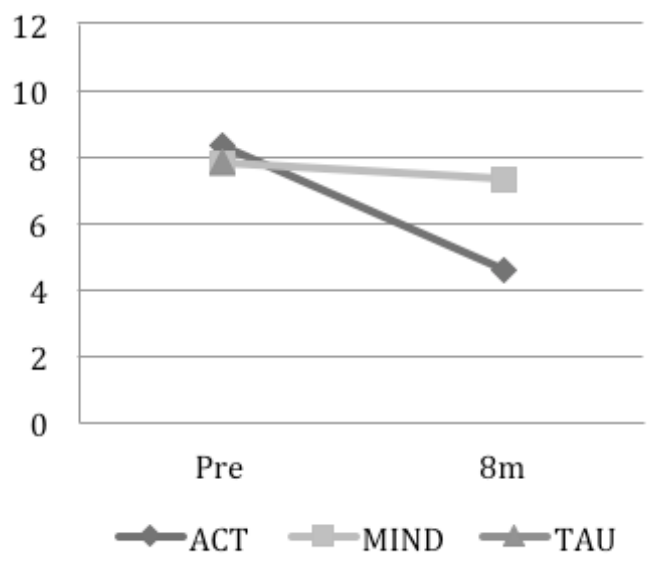

Psicoticismo

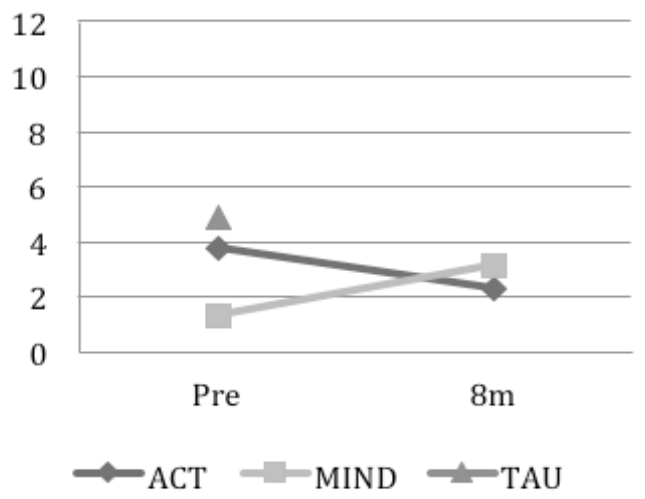

Figura 2. Variables de resultado.

\section{Depresión}

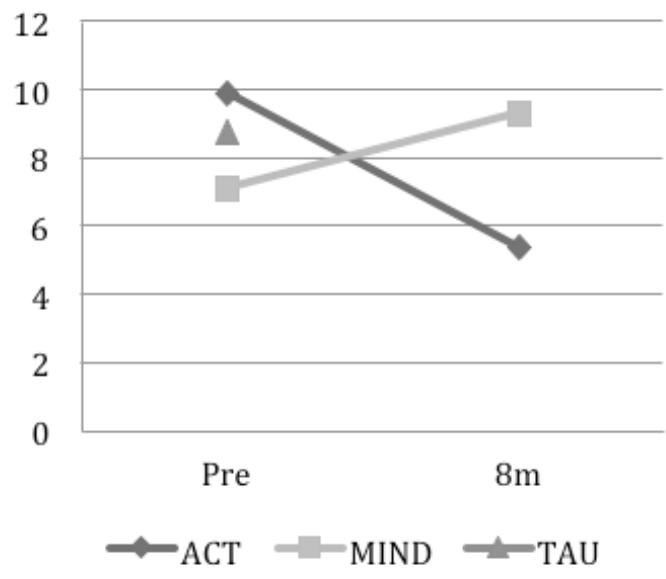

Ideación paranoide

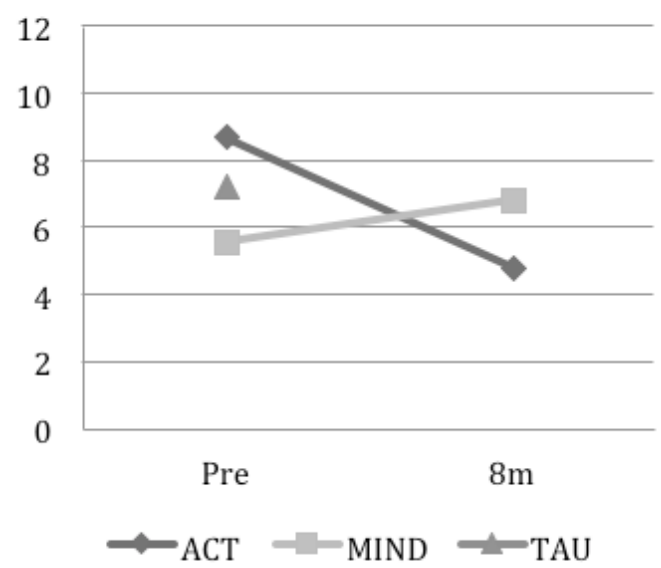


En contraste, la inflexibilidad psicológica en el grupo ACT decreció gradualmente desde el pre-test hasta el seguimiento a los 8 meses: hubo una reducción estadísticamente significativa a lo largo de las cuatro mediciones ( $F$ $(3,27)=6.543, p=.002, \eta 2=.421)$. Además, se encontraron cambios significativos entre el pre-test y seguimiento a los 5 meses $(F(1,10)$ $=6.149, \mathrm{p}=.033, \mathrm{\eta} 2=.381) \mathrm{y}$ entre el pre-test $\mathrm{y}$ el seguimiento a los 8 meses $(F(1,9)=29.402$, $\mathrm{p}<.001, \mathrm{\eta} 2=.766)$.

No hubo efectos de interacción entre los tres grupos entre el pre-test y el post-test ( $p$ > .050). Sin embargo, hubo un efecto de interacción significativo entre los grupos ACT y MIND entre los seguimientos a los 5 y 8 meses ( $F(1$, $14)=6.998, p=.019 \eta 2=.333$ ). Esto es, mientras que la inflexibilidad psicológica disminuyó en el grupo ACT entre los seguimientos, en el grupo MIND aumentó.

\section{Autorregistro de actividad (AAc)}

La Tabla 2 y la Figura 2 muestran que la actividad autoinformada en el grupo TAU no cambió entre el pre-test y el post-test ( $p>.050)$. En el grupo MIND, se observa un incremento general de la actividad $(F(3,12)=8.000, p=$ $.003, \eta 2=.667)$, la media aumentó significativamente del pre-test al post-test $(F(1,12)=$ 20.892, $p=.001, \eta 2=.635)$ y del post-test al seguimiento a los 5 meses $(F(1,6)=6.250, p$ $=.047, \mathrm{\eta} 2=.510)$. Las diferencias entre el pretest y el seguimiento a los 5 meses $(F(1,6)=$ $21.125, p=.004, \eta 2=.779)$ y entre el pre-test y el seguimiento a los 8 meses $(F(1,5)=7.105$, $\mathrm{p}=.045, \eta 2=.587)$ también fueron significativas. Sin embargo, en este grupo la puntuación de la actividad autoinformada comenzó a decrecer entre los 5 y 8 meses de seguimiento.

De manera similar, hubo un incremento significativo general entre las cuatro mediciones en el grupo $\operatorname{ACT}(F(3,24)=22.621, p<.001, \eta 2$ $=.739$ ), que también resultó significativa entre el pre-test y el post-test $(F(1,10)=32.232$, $p<$ $.001, \eta 2=.763)$ y entre el post-test y el seguimiento a los 5 meses $(F(1,9)=6.612, p=.001$, $\eta 2=.424)$. Las comparaciones con el pre-test también fueron significativas, tanto a los 5 meses de seguimiento $(F(1,9)=92.842, p<.001$, $\eta 2=.912)$ como a los 8 meses de seguimiento $(F(1,10)=105.946, p<.001, \eta 2=.914)$.

Comparando los grupos, entre el pre-test y el post-test hubo un efecto de interacción significativo $(F(2,31)=8.194, p=.001, \eta 2=.346)$.

Hubo una interacción significativa entre las condiciones TAU y MIND $(F(1,21)=10.135, p$ $=.004, \eta 2=.326)$ y entre las condiciones ACT y $\operatorname{TAU}(F(1,19)=19.704, \mathrm{p}<.001, \eta 2=.509)$ pero no entre las condiciones ACT y MIND en el post-test. Así, los autorregistros de actividad crecieron en los grupos ACT y MIND, pero no el en grupo TAU. No hubo interacciones entre las condiciones ACT y MIND ( $p>.050)$.

\section{Informes de actividad (IAC)}

Tal y como se refleja en la Tabla 1 y la Figura 2, los informes de actividad del personal hospitalario no cambiaron en el grupo TAU, pero mostraron un ligero incremento en los otros dos grupos entre el pre-test y el post-test. Sin embargo, en el grupo MIND, los informes de actividad del personal hospitalario empezaron a decrecer a lo largo de los dos seguimientos, mientras que en el grupo ACT siguieron cre- 
ciendo, pero no de forma estadísticamente significativa $(p>.050)$. No se encontraron efectos de interacción ( $p>$.050) entre las condiciones ACT y MIND.

El análisis ANOVA mostró que hubo diferencias significativas en el post-test entre grupos $(\mathrm{F}(2,27)=5.205, \mathrm{p}=.012, \eta 2=.278)$. Las comparaciones post hoc indicaron que los grupos MIND y TAU (t $(20)=3.202, \mathrm{p}=.004, \mathrm{~d}=3.20$, MIND: $\mathrm{M}=7.36, \mathrm{SD}=.92, \mathrm{TAU}: \mathrm{M}=5.73, \mathrm{SD}=$ 1.42) y los grupos ACT y MIND ( $t(17)=2.548, p$ $=.021, \mathrm{~d}=2.55$, MIND: $\mathrm{M}=7.36, \mathrm{SD}=.92, \mathrm{ACT}$ : $M=6.00, S D=1.41)$ fueron significativamente diferentes. Los participantes en el grupo MIND mostraron mayor actividad (según los informes del personal hospitalario) que los otros dos grupos en el post-test. Sin embargo, la diferencia entre ACT y MIND desapareció a lo largo de los seguimientos, viéndose que a los 8 meses, los informes de actividad del personal hospitalario para el grupo MIND se redujeron mientras que siguieron creciendo lentamente en el grupo ACT. A pesar de esta tendencia, no se encontraron diferencias entre ambos gru$\operatorname{pos}(\mathrm{ACT}: \mathrm{M}=7.00, \mathrm{SD}=.82$; MIND: $\mathrm{M}=7.00$, $\mathrm{SD}=1.41)$.

\section{Síntomas psicopatológicos (SA-45)}

Se administró el SA-45 a todos los grupos en el pre-test y después a los grupos ACT y MIND en el seguimiento a los 8 meses. Así, el análisis se centró en las diferencias entre los grupos ACT y MIND entre el pre-test y el seguimiento.

\section{Depresión}

Los análisis intragrupales en el grupo ACT indicaron un descenso significativo respecto del pre-test en el seguimiento a los 8 meses
$(\mathrm{F}(1,9)=8.341, \mathrm{p}=.018, \mathrm{\eta} 2=.481)$, mientras que en el grupo MIND las puntuaciones en depresión aumentan. Sin embargo, no se encontraron efectos de interacción.

\section{Ansiedad}

Los niveles de ansiedad se redujeron tanto en el grupo MIND como en el grupo ACT: el análisis intragrupal en el grupo ACT mostró un descenso significativo, $F(1,9)=7.078, p=.026$, $\eta 2=.440)$, mientras que en el grupo MIND, los descensos en los niveles de ansiedad no alcanzaron un nivel estadísticamente significativo. No se encontró interacción ( $p$ > .050).

\section{Ideación paranoide}

Los niveles de ideación paranoide se redujeron significativamente en el grupo ACT (F (1, 9) $=7.024, p=.026, \eta 2=.438)$. Sin embargo la ideación paranoide aumentó en el grupo MIND. No se encontró interacción.

\section{Psicoticismo}

Los niveles de psicoticismo se redujeron muy ligeramente en el grupo ACT e incrementaron en el grupo MIND, pero estos cambios no alcanzaron un nivel estadísticamente significativo y no se encontró interacción ( $p$ > .050).

\section{Tamaños de efecto}

La Figura 3 muestra los tamaños de efecto ( " $d$ " de Cohen) para los tres grupos a lo largo de tres momentos. Las puntuaciones positivas indican efectos en la dirección esperada en comparación con pre-test, mientras que, las negativas indican cambios en dirección contraria. 
La figura 3 muestra que en el grupo TAU, los tamaños de efecto nunca fueron grandes $(.5<\mathrm{d}<.8)$.

En el grupo ACT (parte inferior de la Figura $3)$, tres puntuaciones " $d$ " de Cohen indicaron que hubo un pequeño efecto $(.2<d<.5)$, tres un efecto moderado $(.5<\mathrm{d}<.8)$ y 7 un efecto grande $(\mathrm{d}>$.8). Los tamaños de efecto grandes se encontraron en el AAQ-II, los registros de actividad, y las escalas SA-45 de depresion e ideación paranoide.

En el grupo MIND, cinco puntuaciones " $d$ " de Cohen indicaron que no hubo un efecto de intervención clínicamente relevante $(d<.2)$, dos indicaron que hubo un pequeño efecto $(.2<\mathrm{d}<.5), 4$ un efecto moderado $(.5<\mathrm{d}<.8)$ y 2 un efecto grande ( $d>.8)$. Los tamaños de efecto grandes se encontraron en los registros de actividad.

En el seguimiento a los 8 meses, se observaron efectos grandes en 4 medidas en el grupo ACT (AAQ-II, autorregistro de actividad y escalas SA-45 de depresión e ideación paranoide), en comparación con ninguna medida en el grupo MIND.

\section{Discusión}

Este estudio intenta dar respuesta a una serie de limitaciones tradicionalmente asociadas a las investigaciones e intervenciones tanto farmacológicas como psicosociales actuales. En primer lugar, se enfatizó el funcionamiento social mediante la utilización de registros de actividad y de acciones en dirección de valor. En segundo lugar, se valoraron los resultados a largo plazo (seguimientos a los 5 y 8 meses) para valorar el resultado de las intervenciones más allá del periodo de intervención. En tercer lugar, se tuvieron en cuenta no sólo los efectos directos de la intervención, sino también los efectos indirectos sobre la vida de las personas. Y en último lugar, se utilizaron criterios de inclusión no basados en diagnósticos formales permitiendo una mayor generalización de los resultados a la práctica clínica habitual.

La presente investigación es el primer intento por comparar protocolos ACT y MIND con personas con trastornos mentales graves y cronificados, estando la mayoría de ellos residiendo en instituciones de salud mental. Se empleó una condición TAU para controlar los efectos de ambos protocolos después del tratamiento. A continuación procedemos a realizar un resumen de los datos, tras lo cual expondremos los resultados más importantes, así como los factores a favor y limitaciones del estudio.

Dado el apoyo empírico de la ACT en pacientes con psicosis así como los datos, más limitados pero aún así esperanzadores, que apoyan la eficacia del Mindfulness, no se predijeron diferencias en la efectividad global de ambos protocolos en el post-test. Sin embargo, en vista de la evidencia de que algunos de los efectos en estos estudios de ACT en psicosis pueden ser relativamente duraderos y de acuerdo con el énfasis de ACT en el proceso de vivir de manera consistente con los propios valores, propusimos que los tamaños de efecto serían mayores en este grupo en el seguimiento

Cabe destacar el alto porcentaje de pacientes que completaron el protocolo hasta el final (75 y $81 \%$ respectivamente, -12 de 16 - en ACT y -13 de 16- en MIND). La permanencia de 

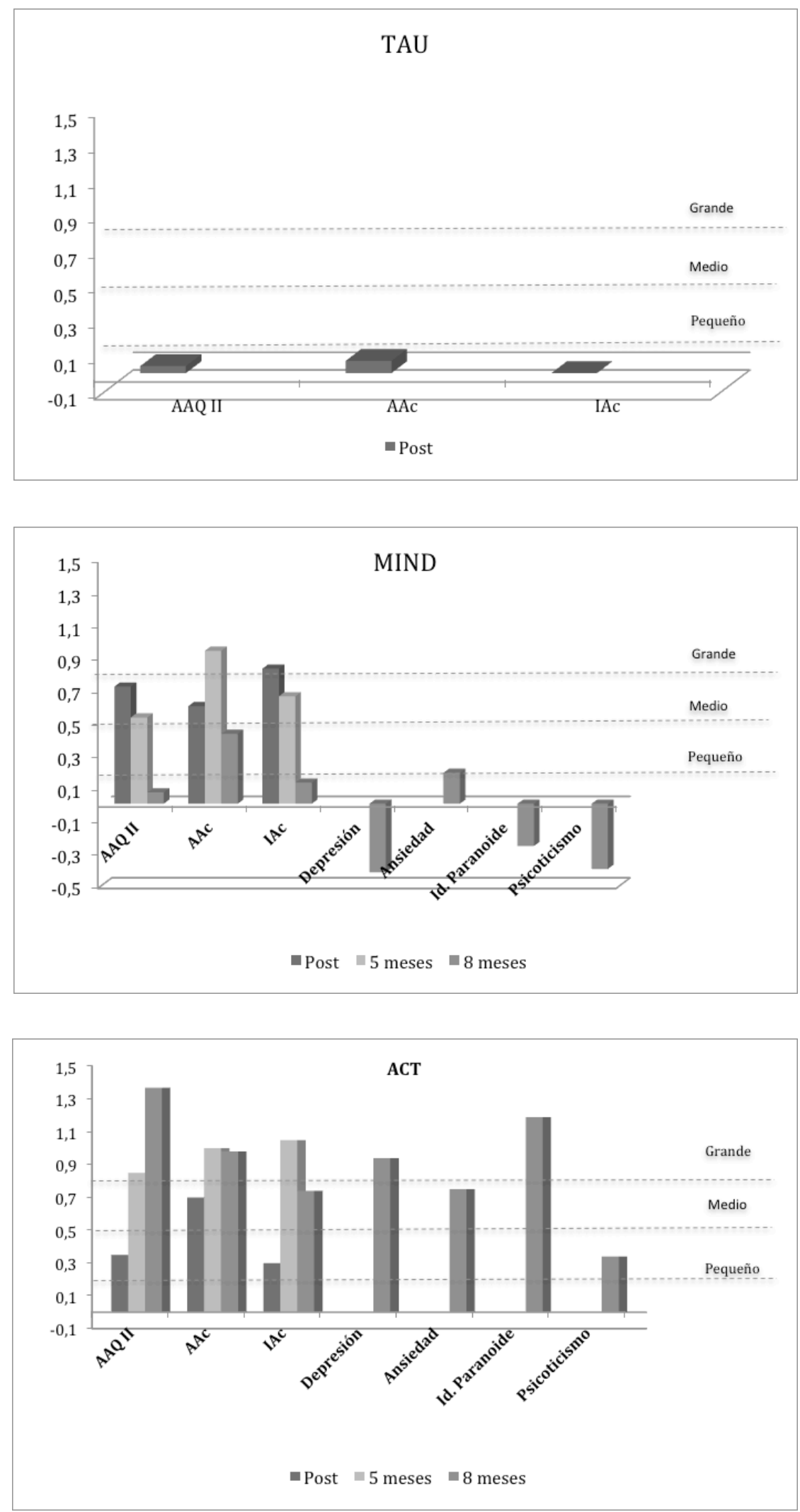

Figura 3. Condiciones TAU, MIND y ACT : tamaños del efecto ("d" de Cohen) intracondición de todas las medidas. Los valores positivos indican efectos en la dirección esperada en comparación con pre-test, los valores negativos indican cambios en dirección contraria a la esperada. 
los participantes a lo largo de la intervención puede estar relacionada con el contexto motivacional que promovieron ambos protocolos. Por ejemplo, el protocolo ACT enfatizó el papel de la elección personal tanto para recibir la implementación del protocolo, como para elegir la dirección de los cambios que perseguían. Además, se les dieron varias oportunidades para elegir si hacer o no los ejercicios, así como si usarlos o no fuera del contexto de la terapia. Esto resulta ser lo contrario de lo que ocurre en los protocolos más habituales, donde se les dice a los pacientes lo que deberían hacer y se dirigen pocos esfuerzos a establecer un contexto que permita a los pacientes tomar decisiones.

Al post-test, las condiciones ACT y MIND mostraron cambios positivos similares y mayores que la condición TAU en la mayoría de las medidas. Las puntuaciones en el AAQ-II decrecieron en ambas condiciones ACT y MIND (aunque más en la segunda), en comparación con la ausencia de cambio en las puntuaciones en TAU. Sin embargo, los cambios no fueron significativos. Las reducciones en las puntuaciones en el $A A Q$ en el post-test son coherentes con estudios previos en ACT (White et al., 2011).

Sí se encontraron diferencias estadísticamente significativas respecto a los incrementos en los autorregistros de actividad. Los resultados obtenidos sugieren que ambos protocolos actuaron de manera similar y tuvieron efecto en medidas tales como la perspectiva del participante respecto de su propia conducta y la perspectiva del personal hospitalario de la conducta de los participantes. Estas medidas no se han tenido en cuenta habitual- mente en estudios previos que, por lo general, se centraron únicamente en medidas formales. Sin embargo, disponer de información acerca de la conducta de los pacientes en el contexto habitual y de cómo los participantes discriminan su propio comportamiento parece ser importante a la hora de validar los protocolos de tratamiento.

A largo plazo solamente pudieron compararse las condiciones ACT y MIND. Si se toman los seguimientos en su conjunto, se observa en primer lugar que los cambios obtenidos al post-test en AAQ/ inflexibilidad mostraron diferentes evoluciones en los seguimientos. Los pacientes en la condición ACT mantuvieron la tendencia al alza en flexibilidad, es decir, la mayoría de ellos redujeron sus puntuaciones en evitación. En contraste a los cambios positivos en el grupo ACT, los pacientes en el grupo MIND mostraron la tendencia opuesta tras el post-test. Esto es, después del post-test, las puntuaciones en el AAQ-II aumentaron en los pacientes del grupo MIND. Este resultado se muestra coherente con estudios previos en ACT que obtuvieron cambios en el AAQ-II pero no inmediatamente después de la implementación del protocolo (véase White et al., 2011). Una razón posible es que los cambios en las puntuaciones del AAQ pueden tomar algo de tiempo, siendo necesarias múltiples situaciones en las que el paciente actúe de acuerdo a una dirección en particular mientras el malestar o el dolor está presente. Consecuentemente, los estudios que proporcionan pocas oportunidades para comportarse de manera flexible no incluyen ni recogen los cambios acumulativos que ocurren al tener múltiples 
oportunidades. Respecto al protocolo MIND y el incremento de las puntuaciones en el AAQII durante el seguimiento, no puede realizarse una comparación con estudios previos porque aquellos que se realizaron con población similar no recogieron medidas de seguimiento (Chadwick et al., 2009; Langer et al., 2012).

En cuanto a los informes del personal hospitalario, reflejan que la actividad continuó mejorando en los pacientes ACT, mientras que comenzó a reducirse en los pacientes MIND. Asimismo, los niveles de depresión, ansiedad e ideación paranoide se redujeron significativamente en el grupo ACT para el seguimiento a los 8 meses, mientras que aumentó o se mantuvo en el grupo MIND en el mismo periodo. También se observaron reducciones significativas en sintomatología depresiva en estudios anteriores sobre ACT con participantes similares, realizados por Gaudiano y Herbert (2006), Zettle y Petersen (2009) y White, et al. (2011). Cabe destacar que el descenso en medidas tales como ansiedad, depresión o ideación paranoide se dieron a pesar de no ser un objetivo de la intervención ACT. El hecho de que estos mismos resultados no se obtuvieran en el mismo periodo de tiempo en la condición MIND es coherente con estudios previos donde se implementaron protocolos de Mindfulness. Así, Chadwick, Hughes, Russell, Russell y Dagnan (2009) no encontraron cambios en la Escala para la Valoración de Síntomas Psiquiátricos (PSYRATS). Un motivo para que se produjese el cambio que tuvo lugar en la condición $A C T$, pero no en la condición MIND, es que en la primera se realizan acciones elegidas libremente por el paciente donde la sintomatología problemática está presente. Sin embargo, en el protocolo MIND no se hizo referencia explícita acerca del papel de los síntomas problemáticos en la realización de acciones importantes para la vida de la persona.

A modo de resumen, la tendencia a la mejora en la condición ACT continuó a lo largo del seguimiento, mientras que la tendencia cambió en los pacientes en la condición MIND. De esta forma, ambos protocolos MIND y ACT fueron efectivos en el post-test, pero sólo el protocolo ACT mantuvo y mejoró los efectos en el seguimiento a los 8 meses. De manera general, puede concluirse que ambos protocolos actuaron de manera similar a corto plazo pero no a largo plazo. Existen varias razones para entender los resultados positivos en el seguimiento en la condición ACT, así como el desvanecimiento en el seguimiento de los resultados positivos en la condición MIND.

Una razón central es que las intervenciones basadas en ACT están focalizadas directamente en la reducción de patrones de regulación basados en la evitación experiencial mientras se notan los pensamientos, recuerdos y emociones desagradables que previamente pudieran estar funcionando como una barrera a la hora de actuar de acuerdo a los valores personales. Sin embargo, los protocolos basados en Mindfulness pueden hacer esto mismo de una manera indirecta, es decir, al implementarse un protocolo MIND, pueden darse diferentes efectos en las acciones en dirección de valor en función de la historia personal de cada uno. Esto no es así en el caso del protocolo ACT, diseñado para hacer explícita la dirección de las 
interacciones de defusión/Mindfulness. Respecto al protocolo ACT, los cambios obtenidos en el patrón de evitación experiencial son bastante similares a los obtenidos en otros estudios, por ejemplo, con adolescentes con depresión, fobia social y con pacientes psicóticos (Hayes et al., 2011; Ossmand et al., 2006; White et al., 2011).

Es importante mencionar que, aunque ambos protocolos están diseñados para enseñar a los pacientes a interactuar de manera similar con el malestar y los pensamientos problemáticos sin intentar controlarlos, el protocolo ACT se focaliza explícitamente en la clarificación de las direcciones de valor, mientras que el protocolo MIND no explicita necesariamente los contextos de valor y por tanto la experiencia de malestar no se pone siempre al servicio de una vida significativa a lo largo del entrenamiento. Esta última puede ser la razón de las diferencias entre ambos protocolos. En dicho contexto, los pacientes en la condición MIND pueden haber usado el entrenamiento en Mindfulness como una forma de aprender cómo controlar el malestar, aun habiéndose dejado claro desde un principio que éste no era el objetivo. Es posible que el aumento de la actividad al post-test en el grupo Mindfulness, nosedebaa la relación deinclusión del malestar y las direcciones valoradas, sino a una relación de oposición donde la barrera (malestar) pudiera ser manejable a corto plazo mientras el participante aprendía estrategias MIND. De acuerdo con esto, los resultados en el grupo ACT podrían reflejar un cambio en el valor funcional del malestar, desde ser opuesto a las acciones valoradas, a estar incluido como parte de ellas. Así, lo fundamental serían las acciones de acuerdo a valores, conjuntamente con la práctica de defusión para establecer una relación jerárquica entre el self y los pensamientos/sentimientos (Luciano et al., 2011).

Para concluir, a pesar de que ACT incluye muchos elementos de Mindfulness, ambas intervenciones no llegan a ser equivalentes. ACT tiene el componente añadido del contexto de valor personal que parece constituir un elemento distintivo clave entre ambos protocolos.

De la presente investigación se derivan además las siguientes implicaciones. En primer lugar, se observa de forma positiva la posibilidad de medir la eficacia de una intervención en base a la realización de acciones comprometidas con direcciones significativas para la persona, más que en base a la reducción sintomática. En segundo lugar, las intervenciones basadas en ACT encajan a la perfección en la perspectiva de la rehabilitación, incorporando además del contexto de valor, el trabajo para la distinción entre uno mismo y los eventos privados. En tercer lugar, las intervenciones basadas en lo importante para la persona, pueden mejorar la gestión de recursos, ya que favorecen su adherencia. Si añadimos que el protocolo realizado en la investigación resultó muy breve (2 meses), se puede derivar que la intervención ha presentado un importante nivel de eficiencia. En cuarto lugar, estamos ante una propuesta no directiva que potencia la responsabilidad de las elecciones personales y además consigue desestigmatizar realmente los problemas mentales al liberar a éstos del control de la conducta. En quinto lugar, el he- 
cho de haber utilizado terapeutas inexpertos hace pensar que con terapeutas con mayor experiencia clínica se podrían esperar mejores resultados. Y finalmente, se resta relevancia al diagnóstico a la hora de abordar la intervención de personas con Trastorno Mental Crónico, demostrando que la dimensión funcional de la Evitación Experiencial Destructiva es una alternativa a los sistemas diagnósticos tradicionales, que permite abordar desde el punto de vista funcional gran cantidad de trastornos y que posibilita que las intervenciones grupales no requieran necesariamente homogeneidad entre sus miembros.

Como se ha dicho, el presente estudio no carece de limitaciones. Principalmente, la pérdida de datos a lo largo del proceso tanto respecto de los datos obtenidos directamente de los pacientes como de los obtenidos del personal hospitalario. Esto debería servir para reclamar una gestión diferente de la investigación en el hospital. Además, es una pena no haber podido mantener la condición de control a lo largo del seguimiento por razones éticas, que insta a pensar en nuevas y creativas formas de obtener los resultados sin estos problemas. Así pues, se necesita más investigación para mejorar los aspectos señalados hasta ahora, así como para aumentar el tamaño de la muestra, mejorar las medidas de las variables de respuesta, reducir la pérdida de datos a lo largo del seguimiento, incorporar sesiones grabadas en vídeo, así como mejorar el entrenamiento a terapeutas y el ajuste de protocolos a la investigación reciente.

Como conclusión, los resultados obtenidos con pacientes con problemas mentales crónicos parecen apoyar la efectividad de las intervenciones en Terapia de Aceptación y Compromiso centradas en una potenciación no directiva de la responsabilidad de las personas a la hora de tomar sus propias decisiones mientras aprenden una manera alternativa de relacionarse con pensamientos, emociones y recuerdos displacenteros. Dicho de otra forma, aprender a relacionarse con los componentes de uno mismo con la perspectiva de inclusión/ aceptación en lugar de perder tiempo de la propia vida en tratar de eliminar o controlar los pensamientos o sentimientos.

\section{Referencias}

Abba, N., Chadwick, P., \& Stevenson, C. (2008). Responding mindfully to distressing psychosis: A grounded theory analysis. Psychotherapy Research, 18, 77-87.

Bach, P. \& Hayes, S. C. (2002). The use of acceptance and commitment therapy to prevent the rehospitalization of psychotic patients: a randomized controlled trial. Journal of Consulting and Clinical Psychology, 70, 1129-1139.

Bach, P., Hayes, S. C., \& Gallop, R. (2012). Long term effects of brief acceptance and commitment therapy for psychosis. Behavior Modification, 36, 165-181.

Bond, F. W., Hayes, S. C., Baer, R. A., Carpenter, K. M., Guenole, N., Orcutt, H. K., Waltz, T., \& Zettle, R.D. (2011). Preliminary psychometric properties of the Acceptance and Action Questionniare - II: A revised measure of psychological flexibility and experiential avoidance. Behavior Therapy, 42, 676-688.

Chadwick, P., Newman-Taylor, K., \& Abba, N. (2005). Mindfulness groups for people with distressing psychosis. Behavioural and Cognitive Psychoterapy, 33, 351-359.

Chadwick, P., Hughes, S., Russell, D., Russell, I., \& Dagnan, D. (2009). Mindfulness groups for distressing voices and paranoia: a replication and randomized feasibility trial. Behavioural and Cognitive Psychotherapy, 37, 403-412. 
Cohen, J. (1988). Statistical power analysis for the behavioral sciences (2nd Ed.) Hillsdale, NJ: Erlbaum.

Davison, M., Bershadsky, B., Bieber, J., Silversmith, D., Maruish, M., \& Kane, R. (1997). Development of a brief, multidimensional, self-report instrument for treatment outcomes assessment in psychiatric settings: Preliminary findings. Assessment, 4, 259-276.

Derogatis, L.R. (1994). Symptom Checklist90-R (SCL-90-R): Administration, scoring and procedures manual (3rd ed.). Minneapolis: NCS Pearsons, Inc

Endicott, J., Spitzer, R., Fleiss, J., \& Cohen, J. (1976). The global assessment scale: A procedure for measuring overall severity of psychiatric disturbance. Archives of General Psychiatry, 33, 766-771.

Gaudiano, B. A., \& Herbert, J. D. (2006). Acute treatment of inpatients with psychotic symptoms using Acceptance and Commitment Therapy: Pilot results. Behaviour Research and Therapy, 44, 415437.

Hayes, S. C., Barnes-Holmes, D., \& Roche, B. (2001). Relational Frame Theory: A postSkinnerian account of human language and cognition. New York: Springer.

Hayes, L., Boyd, C., \& Sewell, J. (2011). Acceptance and Commitment Therapy for the treatment of adolescent depression: A pilot study in a psychiatric outpatient setting. Mindfulness, 2, 86-94. doi: 10.1007/ s12671-011-0046-5

Hayes, S. C., Luoma, J. B., Bond, F. W., Masuda, A., \& Lillis, J. (2006). Acceptance and commitment therapy: model, processes and outcomes. Behaviour Research and Therapy, 44, 1-25.

Hayes, S. C., \& Smith, S. (2005). Get out of your mind and into your life. The new Acceptance and Commitment Therapy. Oakland: New Harbinger Publications, Inc.

Hayes, S. C., Strosahl, K., \& Wilson, K. (1999). Acceptance and Commitment Therapy: An experiential approach to behavior change. New York: Guilford Press.

Hayes, S. C., Strosahl, K., Wilson, K. G., Bissett,
R. T., Pistorello, J., Toarmino, D.,... McCurry, S. M. (2004). Measuring experiential avoidance: a preliminary test of a working model. Psychological Record, 54, 553-578.

Kabat Zinn, J. (1990). Vivir con plenitud las crisis. Barcelona: Kairós.

Kabat-Zinn, J. (2009). Mindfulness en la vida cotidiana. Donde quiera que vayas, ahí estás. Barcelona: Paidós.

Langer, A. I., \& Cangas, A. J. (2007). Fundamentos y controversias en la diferenciación entre alucinaciones en población clínica y normal. Terapia Psicológica, 25, 173-182.

Langer, A. I., Cangas, A. J., Salcedo, E., \& Fuentes, B. (2012). Applying mindfulness therapy in a group of psychotic individuals: A controlled study. Behavioural and Cognitive Psychotherapy, 40, 105-109.

Luciano Soriano, M. C., \& Hayes, S. C. (2001). Trastorno de Evitación Experiencial. Revista Internacional de Psicología Clínica y de la Salud, 1, 109-157.

Malone, D., Newron-Howes, G., Simmonds, S., Marriot, S., \& Tyrer, P. (2007). Community mental health teams (CMHTs) for people with severe mental illnesses and disordered personality.CochraneDatabaseofSystematic Reviews, 3. doi: 10.1002/14651858. CD000270.pub2

National Institute of Mental Health (1987). Towards a Model for Comprehensive Community-Based Mental Health System. Washington, DC: NIMH.

Ruiz, F. J., Langer Herrera, A. I., Luciano, C., Cangas, A. J., \& Beltran, I. (2013). Measuring experiential avoidance and psychological inflexibility: The Spanish version of the Acceptance and Action Questionnaire II. Psicothema, 25, 123-129. doi: 10.7334/ psicothema2011.239

Ruiz, F. J., \& Luciano C. (en prensa). Improving international level chess-players' performance with an acceptance-based protocol: Preliminary findings. The Psychological Record.

Salgado, C. F., Luciano, C., \& Gutiérrez, O. (2013). Eficacia de la Terapia de Aceptación y Compromiso en personas con Trastorno Mental Crónico. Un ensayo aleatorizado. 
Informaciones Psiquiátricas, 214, 385-401.

Sandin, B., Valiente, R. M., Chorot, P., Santed,

M. A., \& Lostao, L. (2008). SA-45: forma abreviada del SCL 90. Psicothema, 20, 290296.

Segal, Z. V., Williams, J. M., \& Teasdale, J. D. (2002). Mindfulness-based cognitive therapy for depression. New York: Guilford Press.

White, R., Gumley, A., McTaggart, J., Rattrie, L., McConville, D., Cleare, S., \& Mitchell, G. (2011). A feasibility study of Acceptance and Commitment Therapy for emotional dysfunction following psychosis. Behaviour Research and Therapy, 49, 901-907.

Williams, J., Teasdale, J., Segal, Z., \& Soulsby, J. (2000). Mindfulness-based cognitive therapy reduces overgeneral autobiographical memory in formerly depressed patients. Journal of Abnormal Psychology, 109, 150-155.

Wilson, K. G., \& Luciano Soriano, M. C. (2002). Terapia de Aceptación y Compromiso (ACT). Un tratamiento conductual orientado a los valores. Madrid. Ed. Pirámide. 